\title{
Authors' reply: influenza vaccine effectiveness: heterogeneity in estimates for the 2012/13 season
}

K Mølbak (krm@ssi.dk)1, H D Emborg ${ }^{1}$, S Gubbels¹, K Bragstad², T G Krause ${ }^{1}$, T K Fischer ${ }^{2}$

1. Department of Infectious Disease Epidemiology, Statens Serum Institut, Copenhagen, Denmark

2. National Influenza Centre, Department of Microbiological Diagnostics and Virology, Statens Serum Institut, Copenhagen, Denmark

Citation style for this article:

Mølbak K, Emborg HD, Gubbels S, Bragstad K, Krause TG, Kølsen Fischer T. Authors' reply: Influenza vaccine effectiveness: heterogeneity in estimates for the 2012/13 season. Euro Surveill. 2013;18(7):pii=20401. Available online: http://www.eurosurveillance.org/ViewArticle.aspx?Articleld=20401

Article published on 14 February 2013

To the editor: We thank Wim van der Hoek and colleagues for their interest in our work [1] and for sharing the preliminary data on influenza vaccine effectiveness (VE) for the $2012 / 13$ season in the Netherlands. When we wrote our article, we emphasised that the findings were based on analysis of data from elderly people mainly admitted to hospital. We cannot make any inference as regards VE among younger, unselected individuals seen in primary healthcare. However, the main public health objective with the influenza vaccination campaign is to prevent morbidity and premature mortality among the frail and the elderly. With this in mind, our observations do have relevance.

We found a low VE of $-11 \%$ ( $95 \%$ confidence interval: $-41 \%$ to $14 \%$ ) against influenza A among patients 65 years and older. This is not a stand-alone observation but is corroborated by other lines of evidence. In the period we studied, influenza $\mathrm{A}\left(\mathrm{H}_{3} \mathrm{~N}_{2}\right)$ was the dominant influenza subtype in Denmark, and genetic characterisation of the strain revealed a clade that, compared with vaccine strain A/Victoria/361/2011, contained seven amino acid substitutions in the haemagglutinin (HA) gene. It is possible that widespread circulation in Denmark of this clade may be a biological explanation of the disappointing VE against influenza A. In our paper, we mention that this clade was demonstrated in patients who had been vaccinated with seasonal influenza vaccine. Further support to our findings is provided from registration of influenza cases in Danish intensive care units (ICU). Current data suggest that 22 of 58 patients admitted with influenza $A$ this season had been vaccinated, corresponding to $38 \%$ vaccine failures. In the three previous seasons, the proportion of vaccine failures among patients in ICUs ranged between $15 \%$ and $19 \%$. Finally, an estimated VE against influenza B of $69 \%$ renders credibility to our epidemiological methodology and data.

We agree with van der Hoek et al. that it is important to consider sources of bias and confounding. It is argued that patients with underlying medical conditions are more likely to seek medical care in case of acute febrile illness, more likely to be admitted to hospital, and more likely to get an influenza diagnostic laboratory test. We consider these arguments reasonable. Age can be regarded as a crude proxy of comorbidity. In the study period, the national testing rate for influenza was age-dependent and ranged from 119 per 100,000 population in $65-69$ year-olds to 179 per 100,000 among patients 80 years and older (Table). Indirectly, this suggests that the very old and frail are tested more often than the healthy seniors, as argued above. The advantage of the test-negative design is that both cases and controls are subject to the same selection process (i.e. seeking medical care and being tested)

\section{TABLE 1}

National testing activity for influenza, number and proportion of samples tested positive for influenza A, and age-specific crude vaccine effectiveness estimated on the figures provided in [1]

\begin{tabular}{|l|c|c|c|c|c|c|}
\hline Age group (years) & Population & Number of tests & $\begin{array}{c}\text { Test rate } \\
\text { per 100,000 } \\
\text { population }\end{array}$ & $\begin{array}{c}\text { Number positive } \\
\text { for influenza A }\end{array}$ & $\begin{array}{c}\text { Proportion of all } \\
\text { tests positive for } \\
\text { influenza A } \\
(\%)\end{array}$ & $\begin{array}{c}\text { Vaccine effectiveness in age } \\
\text { strata } \\
\%(95 \% \text { CI) }\end{array}$ \\
\hline $65-69$ & 350,623 & 416 & 119 & 75 & 63 & $15.1 \%(-44.9$ to 50.3) \\
\hline $70-74$ & 239,485 & 321 & 134 & 70 & 52 & $1.7 \%(-70.2$ to 43.3$)$ \\
\hline $75-70$ & 171,586 & 290 & 169 & 84 & 50 & $-29.3 \%(-116$ to 22.3) \\
\hline$\geq 80$ & 232,821 & 416 & 179 & 135 & 76 & $-24.9 \%(-89.0$ to 17.4$)$ \\
\hline
\end{tabular}

$\mathrm{Cl}$ : confidence interval.

a Danish population data from www.statistikbanken.dk, last quarter of 2012. 
and therefore the concerns raised by van der Hoek are to a large extent covered by the analytical design.

Van der Hoek et al. argue further that a larger proportion of influenza virus infections would be detected in the vaccinated group compared to the non-vaccinated group. This would only be an issue of confounding if both being tested positive and the VE estimate was dependent on comorbidity (e.g. poor VE among those with severe comorbidity). In principle, this cannot be ruled out. Our data do indicate a trend of higher VE estimates in the younger population, but a formal test of heterogeneity of the odds ratio fails to show significant effect-modification $(p=0.66)$.

In conclusion, we consider that the test-negative design combined with the adjustment for age addresses most concerns about bias and confounding, but needless to say, it would be even better to have valid indicators of underlying illness. We note with interest that our Dutch colleagues report a considerable difference between the crude and adjusted VE estimates. It is of methodological interest to learn how much of this adjustment could be accomplished by adjusting for age alone.

For the future we hope to expand our registers to include indicators of underlying illness and to obtain complete and timely data on vaccination uptake among younger individuals. This will allow us to obtain rapid estimates of VE covering a wider range of age groups and potentially also to stratify for different groups of comorbidities. We acknowledge the advantages of VE estimates obtained from networks of primary health physicians, but keeping the objective of the seasonal influenza vaccinations in mind, it is important also to assess the effectiveness among other populations.

Conflict of interest

None declared.

Authors' contributions

$\mathrm{K}$ Mølbak wrote a draft response, H-D Emborg and S Gubbels verified the data and the analysis, T K Fischer, T G Krause and $\mathrm{K}$ Bragstad provided comments, and all authors approved the final version.

References

1. Bragstad K, Emborg HD, Kølsen Fischer T, Voldstedlund M, Gubbels S, Andersen B, Mølbak K, Krause TG. Low vaccine effectiveness against influenza $\mathrm{A}\left(\mathrm{H}_{3} \mathrm{~N}_{2}\right)$ virus among elderly people in Denmark in 2012/13 - a rapid epidemiological and virological assessment. Euro Surveill. 2013;18(6):pii=20397. Available from: http://www.eurosurveillance.org/ViewArticle. aspx?Articleld $=20397$ 